\title{
PESQUISAS DE ENFERMAGEM EM GERONTOLOGIA
}

Karina S. de Almeida Hammerschmidt ${ }^{1}$, Ângela Cristina da Silva Borghi², Maria Helena Lenardt ${ }^{3}$, Márcia Daniele Seima ${ }^{4}$

RESUMO: Trata-se de pesquisa bibliográfica permeada por reflexões teóricas sobre pesquisas de Enfermagem em gerontologia. Teve-se como objetivo identificar a produção científica da Enfermagem em gerontologia. Foram realizadas buscas nas bases de dados LILACS, MedLINE e SciELO e o período pesquisado foi de janeiro de 1990 a janeiro de 2007. Os resultados apontaram 28 publicações, sendo que 20 podem ser classificadas como pesquisa e 8 como reflexão. Dos trabalhos encontrados 26 eram artigos e dois teses; $65 \%$ se apresentavam no idioma inglês e $72 \%$ das publicações foram posteriores ao ano de 2000. Das análises emergiram cinco categorias: geriatria e gerontologia na graduação; prática gerontológica; capacidade funcional de idosos institucionalizados; consulta de enfermagem gerontológica e/ou instrumentos de enfermagem para atenção ao idoso e grupos de estudo/pesquisa em gerontologia. O tema com maior ênfase de trabalhos relaciona-se à prática profissional, cujo foco se refere às patologias que acometem a população idosa, principalmente doenças crônicas.

PALAVRAS-CHAVE: Pesquisa em enfermagem; Gerontologia.

\section{NURSING RESEARCH IN GERONTOLOGY}

\begin{abstract}
This text is about bibliographic research with theoretical reflections about nursing research in gerontology. It aimed to identify the scientific nursing production on gerontology. The searches were performed at LILACS; MedLINE and SciELO databases. The research period was from January 1996 to January 2006. The results included 28 publications, 20 classified as research and 8 as reflections. Among the publications, 26 were articles and 2 were thesis; $65 \%$ were in English and $72 \%$ were released after the year 2000. After analysis we propose five categories: 1) Geriatrics and gerontology in graduation; 2) Gerontology practice; 3) Functional capacity of institutionalized elders; 4) Nursing geriatric consultation and nursing instruments used with the elderly; 5) Study/research groups in gerontology. Most publications focus on professional practice. These publications address elderly people's pathologies mainly chronic diseases.
\end{abstract}

KEYWORDS: Nursing research; Gerontology.

\section{INVESTIGACIONES DE ENFERMERÍA EN GERONTOLOGÍA}

RESUMEN: Esta es una investigación bibliográfica permeada por reflexiones teóricas acerca de las investigaciones de enfermería en gerontología. El objetivo fue identificar la producción científica del oficio de enfermería en gerontología. Fueron realizadas búsquedas en las bases de datos LILACS; MedLINE y SciELO. El periodo de trabajo fue de enero de 1996 a enero de 2007. Los resultados apuntaron 28 publicaciones, siendo que 20 pueden ser clasificadas como investigación y 8, como reflexión. De los trabajos, 26 eran artículos y dos eran tesis; $65 \%$ de ellos están en idioma inglés y $72 \%$ de las publicaciones tienen su fecha después del año 2000 . Dela análisis, siguen cinco categorías: 1) Geriatría y gerontología en la graduación; 2) Práctica gerontológica; 3) Capacidad funcional de ancianos institucionalizados; 4) Consulta de enfermería gerontológica y/o instrumentos de enfermería para atención al anciano; 5) Grupos de estudio/investigación en gerontología. El tema con mayor énfasis de trabajos se relaciona con la práctica profesional. Son investigaciones que plantean la población de ancianos y determinadas patologías, principalmente enfermedades crónicas.

PALABRAS CLAVE: Investigación en enfermería; Gerontología.

${ }^{1}$ Mestranda no Programa de Pós-Graduação em Enfermagem da UFPR. Bolsista do CAPES. Membro do GMPI.

${ }^{2}$ Mestranda no Programa de Pós-Graduação em Enfermagem da UFPR. Bolsista do CAPES. Membro do GMPI.

${ }^{3}$ Doutora em Filosofia da Enfermagem pela UFSC. Professora Sênior do Programa de Pós-Graduação em Enfermagem da UFPR. Coordenadora do grupo Multiprofissional de Pesquisa sobre Idosos - GMPI.

${ }^{4}$ Acadêmica. Curso de Graduação em Enfermagem - UFPR. Bolsista do programa Voluntário de Iniciação Científica - UFPR. Membro do GMPI.

Autor correspondente:

Karina S. de Almeida Hammerschmidt

R. Alferes Poli 271 ap 1503 - 80020-090 - Curitiba-PR

Email: ksalmeidah@yahoo.com.br 


\section{INTRODUÇÃO}

O envelhecimento compreende os processos de transformação do organismo humano, inclui uma desorganização crescente do sistema corporal, determinada por elementos biológicos-genéticos, ecológicos, psicológicos e sócio-culturais, sendo esses aspectos, objetos de discussão em várias ciências ${ }^{(1)}$. Frente às características próprias do envelhecimento, fazem-se necessários trabalhos que envolvam a análise, reflexão e pesquisa com a população idosa, de forma a trazer contribuições à categoria profissional da enfermagem, à população idosa e à sociedade como um todo.

A história mostra, de modo explícito, que a pesquisa no Brasil possui, de forma geral, características de pouca indução e de grande autonomia em relação às necessidades econômicas e sociais do país. No caso da pesquisa em saúde essas características podem ser verificadas com nitidez, conformando certa tradição. Uma perspectiva moderna de Ciência \& Tecnologia (C\&T) em saúde recomenda que esta tradição seja alterada, no sentido de ampliar o espaço de integração e articulação com as necessidades de saúde da população ${ }^{(2: 126)}$.

Acredita-se que a pesquisa em enfermagem gerontológica, em certa proporção, é reflexo do acelerado processo de envelhecimento populacional da atualidade e da preocupação da classe em cuidar do idoso de maneira condizente com suas necessidades. Neste sentido, os membros do Grupo Multiprofissional de Pesquisa sobre Idosos (GMPI) estão compelidos em identificar as tendências das publicações da pesquisa de enfermagem realizadas na área da gerontologia.

Refletir sobre a pesquisa em enfermagem gerontológica possibilita entender a necessidade e a complexidade da relação saber e fazer em enfermagem, e revela os diversos prismas que constituem a teia do cuidado com a população idosa. As pesquisas de enfermagem em gerontologia exigem a predominância de conhecimentos e ações que dêem suporte e subsídios para o suprimento das necessidades dos indivíduos desta faixa etária e contribuam para o conhecimento dos profissionais envolvidos.

É preciso utilizar conhecimentos cujos saberes e significados estejam envoltos na visão pluralista do cuidado, preservando a individualidade e a forma única de relação dialógica entre cuidador e o idoso cuidado. Esta dinâmica evidencia a possibilidade e necessidade de confrontar os aspectos da realidade e da teoria, impulsionando, fortalecendo e incitando a reflexão e a pesquisa com a população idosa.

Diante do exposto, teve-se como objetivo neste trabalho identificar a produção cientifica da enfermagem em gerontologia.

\section{METODOLOGIA}

Trata-se de pesquisa bibliográfica, entremeada por reflexões teóricas sobre as pesquisas de enfermagem em gerontologia. A pesquisa bibliográfica é desenvolvida a partir de material já elaborado, constituído principalmente de livros e artigos científicos e cuja perquirição é realizada por meio da técnica de análise de conteúdo. Tem por finalidade colocar o pesquisador em contato com o que já se produziu a respeito do tema pesquisado ${ }^{(3)}$.

Atentou-se para os seguintes passos ao desenvolver a pesquisa bibliográfica: 1) busca do material nas bases de dados disponíveis e nos catálogos das bibliotecas; 2) seleção dos textos conforme os objetivos; 3) leitura do texto; 4) anotações somente após a leitura crítica do texto; 5) transcrição dos dados exatos e úteis em relação ao tema levantado; 6) registro das idéias críticas ou conjecturas pessoais que emergiram no decorrer da leitura, para posteriores verificações e reflexões; 7) correta citação das fontes no relatório de pesquisa, evitando o problema de uso indevido do material, o que caracteriza a violação das normas nacionais e internacionais de direitos autorais ${ }^{(3)}$.

A busca do material foi realizada no período de 15 de novembro a 20 de janeiro de 2007, utilizandose como palavra chave principal "pesquisa", palavra chave secundária "enfermagem" e palavra chave terciária "gerontologia".

A seleção do material se deu a partir dos seguintes critérios de inclusão: 1- artigos que abordaram explicitamente, em seus resumos, pesquisas de enfermagem em gerontologia; 2 - aqueles que se apresentavam dentro do corte histórico, com o intuito de delimitar o número de dados, publicações realizadas e/ou publicadas no período compreendido de janeiro de 1990 a janeiro de 2007; 3- constar nas bases de dados LILACS (Literatura Latino-Americana e do Caribe em Ciências da Saúde); MedLine (Literatura Internacional em Ciências da Saúde) e SciELO (Scientific Eletronic Library Online).

Ao final da coleta de dados foram selecionados vinte e oito (28) publicações que se adequavam aos objetivos do estudo e, por isso, compuseram o corpus 
de análise $e^{(4)}$.

Para realizar a análise da produção encontrada foi elaborado um instrumento contendo os dados: título, autor, fonte de publicação, ano de publicação, tipo de estudo. Após a leitura minuciosa, os mesmos foram transcritos no roteiro de análise dos dados e cada trabalho foi identificado com um número de um (1) a vinte e oito (28), segundo a ordem das leituras. As bibliografias encontradas foram submetidas à categorização temática.

\section{PESQUISA EM ENFERMAGEM E EM GERONTOLOGIA}

O desenvolvimento de atividade de pesquisa pelo ser humano é motivado, "pela necessidade de pensar, conhecer e expressar-se correta e claramente, utilizando a razão como a capacidade intelectual de olhar e organizar a realidade de modo a torná-la compreensiva"(5). A pesquisa tem sido utilizada como instrumento de construção da realidade e de fundamentação da ciência, no sentido de elucidar, buscar compreensões e soluções aos problemas evidenciados no meio em que vivemos, contribuindo então para a produção de conhecimentos.

Conforme recomendou a $1^{\text {a }}$ Conferência Nacional de Ciência e Tecnologia em Saúde $\left(1^{\text {a }} \mathrm{CNCT} / \mathrm{S}\right)$ realizada em 1994, a política de C\&T em saúde deve ser um elemento constitutivo da política de saúde do país ${ }^{(2)}$. Nesta perspectiva os conhecimentos oriundos das pesquisas podem resultar em melhorias para a vida das pessoas, para os grupos profissionais ou para a comunidade acadêmica desde que sejam reflexos da observação, da ponderação dos problemas apresentados e retornem em resultados às comunidades e grupos estudados. Os conhecimentos da saúde não devem se constituir em segredos corporativistas, mas ser livremente acessíveis a todos.

As publicações são meios para sociabilizar os conhecimentos dos profissionais com seus pares, por meio da leitura, crítica e análise. Ocorre transformação das informações concretizando-se como saber válido que possa instigar outros pesquisadores a repensar conhecimentos. "A incorporação de conteúdos da ciência ao saber cotidiano é um ensinar e aprender a sentir, refletir e pensar esse cotidiano com um novo olhar"().

Para que se alcance o desenvolvimento do conhecimento científico e da competência profissional, o caminho da pesquisa é inevitável. "A atividade de pesquisa é uma das molas propulsoras do avanço do conhecimento, da construção e validação de novas tecnologias e da formação e fortalecimento profissional nas diferentes áreas de atuação e trabalho"(5).

O enfermeiro vem incorporando a pesquisa à sua prática, evidenciado pelo progressivo aumento de revistas especializadas, de cursos de graduação e pós-graduação lato e strictu sens, que estimulam a produção de pesquisas. A maioria da produção científica da enfermagem é proveniente dos cursos de pós-graduação strictu sensu, a partir da década de 80 , e tem permitido avanços na avaliação crítica da prática profissional, estando intimamente ligada a ela ${ }^{(7)}$.

O cenário em que se encontra a pesquisa no Brasil ainda não representa o ideal, visto que a produção internacional apresenta maior expressividade, no entanto progressos têm sido feitos neste sentido. Associa-se ao aumento das produções as diferentes abordagens de pesquisa, ao utilizar-se métodos advindos de outras áreas de conhecimento como as Ciências Sociais e a Antropologia, proporcionando interações interessantes com a área da saúde.

A pesquisa, conectada aos processos de mudança, auxiliam na construção e compreensão das mesmas. Assim, entende-se que possa revelar as modificações resultantes também do aumento da população de idosos no Brasil. Para que se possa compreender e desenhar caminhos de enfrentamento que essa mudança induz é necessário realizar estudos que venham ao encontro das necessidades da população e da comunidade acadêmica.

Frente ao aumento da população de idosos evidenciada nos últimos quarenta anos no Brasil, verificou-se crescente interesse dos profissionais pela gerontologia, principalmente aqueles envolvidos com a docência. Desencadeou-se, ainda, demanda por profissionais capacitados, alicerçados nos fundamentos da gerontologia para que esses sustentem uma prática profissional apropriada às necessidades dos idosos.

Há alguns anos não se concebia, na maioria das escolas de graduação em enfermagem, realizar distinções entre adultos e idosos, fato comprovado pela organização curricular de muitas delas, que não dispensava atenção especial à formação profissional dos acadêmicos em saúde do idoso. Freqüentemente se ministrava a disciplina Saúde do Adulto e do Idoso como única, sem despertar a atenção dos acadêmicos para as peculiaridades fundamentais para desenvolver o cuidado gerontológico.

Saber responder às necessidades desse segmento populacional tem sido uma preocupação de várias profissões e instâncias político-governamentais. No que se refere à enfermagem, desde a década de 70 do século passado, embora de forma isolada, algumas escolas têm 
destinado espaço acadêmico para a prática da assistência, ensino e pesquisa em enfermagem gerontológica e geriátrica $^{(8)}$.

O cuidado de enfermagem se constitui como foco principal da prática destes profissionais e o conhecimento é essencial para o desenvolvimento de ações embasadas e pautadas em literaturas consistentes. Acredita-se que a atenção singular ao idoso venha a contribuir na prevenção de riscos e complicações que este venha a desenvolver, minimizando os impactos que a velhice possa causar em sua vida e preparando-o para vivenciá-la de maneira integral e independente.

O sistema de saúde no Brasil ainda se faz fundamentado no assistencialismo e na medicina curativa, práticas que vêm sendo modificadas com o avanço das pesquisas em gerontologia, as quais apontam para caminhos da prevenção e promoção da saúde, realizadas em bases interprofissionais ${ }^{(9)}$ e na atuação do idoso como protagonista de seu cuidado.

Desenvolver pesquisas gerontológicas torna-se fundamental para solidificar conhecimentos a respeito da população idosa, ao mesmo tempo em que se desenvolve uma cultura de cuidados gerontológicos envolvendo todos os aspectos que possam integrar as várias faces do conhecimento. Sabe-se que o cuidado gerontológico almeja englobar a individualidade, a multidimensionalidade, a ética e a estética, pautado na autonomia do idoso, na promoção da saúde e no incentivo ao envelhecimento ativo e saudável.

Ao se realizar pesquisas gerontológicas, respondese à uma necessidade da sociedade com relação à prevenção, tratamento e cuidado relacionados ao envelhecimento. Assume-se responsabilidades e compromissos com relação ao tema, auxiliando na modificação de idéias preconcebidas sobre o envelhecimento, que o vinculam freqüentemente como sinônimo de doença, perdas e dependência.

O aumento da produção científica em gerontologia indica que modificações se delineiam ao longo do tempo e refletem a preocupação dos profissionais com as temáticas que envolvem o envelhecer e o cuidado gerontológico. Acredita-se que este caminho indique cuidados específicos ao idoso e sejam indispensáveis para a mudança de percepção do envelhecimento.

A pesquisa em enfermagem gerontológica pode evidenciar os caminhos que a comunidade acadêmica tem percorrido, e conhecer esses caminhos contribui para a melhoria da categoria profissional, refletida na qualidade do cuidado e visibilidade da profissão fundamentada em conhecimentos efetivos evidenciados e diagnosticados por meio da pesquisa.

\section{ANÁLISE E DISCUSSÃO DOS RESULTADOS}

Verificou-se inicialmente, durante a coleta dos dados na base LILACS, sete (7) publicações relacionadas à pesquisa/enfermagem/gerontologia, as quais foram publicadas no período de 1990 a 2001. Todas elas foram selecionadas por se enquadrarem nos critérios estabelecidos. Em relação à base de dados SciELO, foram encontradas três (3) publicações relacionadas às palavras pesquisadas (pesquisa/enfermagem/gerontologia) e todas foram utilizadas para análises posteriores. Os trabalhos desta base de dados foram publicados no período de 2002 a 2007.

Na base de dados MedLINE, foram encontrados primariamente vinculados às palavras-chave pesquisa/ enfermagem/gerontologia, cinqüenta e cinco (55) trabalhos, sendo excluídos trinta e sete (37) deles em razão de não privilegiar os requisitos estabelecidos para inclusão, ou por serem idênticos a outros trabalhos já encontrados nas bases de dados LILACS e/ou SciELO. Em síntese, esta base forneceu dezoito (18) trabalhos para o estudo, os quais foram publicados no período de 1996 a 2006.

A seguir, apresenta-se o Quadro 1, com a totalidade dos artigos encontrados nas bases de dados e incluídos no estudo.

Quadro 1 - Publicações utilizadas, segundo bases de dados consultadas. Curitiba, Paraná, 2007

\begin{tabular}{|lcccc|}
\hline & LILACS & SciELO & MedLINE & $\begin{array}{l}\text { Total } \\
\text { de } \\
\text { artigos* }\end{array}$ \\
\hline $\begin{array}{l}\text { Quantidade } \\
\text { de artigos } \\
\text { analisados }\end{array}$ & 7 & 3 & 55 & 28 \\
\hline
\end{tabular}

* Total de publicações utilizadas, excluídos os idênticos ou semelhantes escritos pelos mesmos autores.

Dos vinte e oito (28) textos encontrados, vinte (20) foram classificados como pesquisa e oito (8) na modalidade reflexão e, destes, 26 (vinte e seis) eram artigos e duas (2) publicações na classificação teses.

Apresenta-se, na seqüência, o Quadro 2 que exibe as referências do material selecionado para reflexão deste estudo. 
Quadro 2 - Pesquisas de Enfermagem em Gerontologia. Curitiba, Paraná, 2007

\begin{tabular}{|c|c|c|c|c|}
\hline Nome do Trabalho & Autor & Fonte & $\begin{array}{l}\text { Ano } \\
\text { publicação }\end{array}$ & Tipo de estudo \\
\hline $\begin{array}{l}\text { O ser que envelhece: técnicas, } \\
\text { ciência e saber. }\end{array}$ & $\begin{array}{l}\text { Prado SD, Sayad } \\
\text { JD. }\end{array}$ & Ciência e Saúde Coletiva & 2007 & Reflexão \\
\hline $\begin{array}{l}\text { Perspectivas das pesquisas em } \\
\text { gerontologia e geriatria: } \\
\text { revisão da literatura }\end{array}$ & $\begin{array}{l}\text { Freitas MC, } \\
\text { Maruyama SAT. }\end{array}$ & $\begin{array}{l}\text { Revista Latino Americana } \\
\text { de Enfermagem }\end{array}$ & 2002 & Reflexão \\
\hline $\begin{array}{l}\text { Idosos que foram vítimas } \\
\text { de acidentes de trânsito } \\
\text { no município de Ribeirão Preto - SP, } \\
\text { em } 1998 \text {. }\end{array}$ & $\begin{array}{l}\text { Silveira R, } \\
\text { Rodrigues RAP, } \\
\text { Costa Junior ML. }\end{array}$ & $\begin{array}{l}\text { Revista Latino Americana } \\
\text { de Enfermagem }\end{array}$ & 2002 & Pesquisa \\
\hline $\begin{array}{l}\text { Enfermeira gerontológica: conceptos } \\
\text { para la practica }\end{array}$ & Anzola Perez A. & $\begin{array}{l}\text { Organização Panamericana de } \\
\text { la Salud }\end{array}$ & 1993 & Pesquisa \\
\hline $\begin{array}{l}\text { O enfermeiro como instrumento de ação } \\
\text { no cuidar do idoso: uma relação social } \\
\text { na perspectiva de Alfred Schutz }\end{array}$ & Brum AKR. & $\begin{array}{l}\text { Universidade do Rio de } \\
\text { Janeiro. Faculdade de } \\
\text { Enfermagem }\end{array}$ & 2001 & Pesquisa \\
\hline $\begin{array}{l}\text { Avaliação da capacidade funcional de } \\
\text { idosos institucionalizados }\end{array}$ & Bento MLF. & $\begin{array}{l}\text { Universidade Federal da } \\
\text { Paraiba. Centro de } \\
\text { Ciências da Saúde. } \\
\end{array}$ & 1999 & Pesquisa \\
\hline $\begin{array}{l}\text { Consulta de enfermagem geriátrica e } \\
\text { gerontológica: uma necessidade }\end{array}$ & $\begin{array}{l}\text { Tavares S, Santoro } \\
\text { AC. }\end{array}$ & Acta Paulista Enfermagem & 1999 & Reflexão \\
\hline $\begin{array}{l}\text { A construção da área de gerontologia e } \\
\text { geriatria no Departamento de } \\
\text { Enfermagem da Universidade } \\
\text { Federal de São Paulo: } \\
\text { da concepção a concretização }\end{array}$ & $\begin{array}{l}\text { Bretas ACP, } \\
\text { Yoshitome AY. }\end{array}$ & Acta Paulista Enfermagem & 1998 & Reflexão \\
\hline $\begin{array}{l}\text { Grupos de estudo e pesquisa } \\
\text { em gerontologia que incluem } \\
\text { a enfermagem }\end{array}$ & $\begin{array}{l}\text { Silva EBN, Bretas } \\
\text { ACP. }\end{array}$ & $\begin{array}{l}\text { Texto \& Contexto } \\
\text { Enfermagem }\end{array}$ & 1997 & Pesquisa \\
\hline $\begin{array}{l}\text { Levantamento dos conteúdos de } \\
\text { geriatria e gerontologia dos currículos } \\
\text { dos cursos de graduação em } \\
\text { Enfermagem de Santa Catarina } \\
\text { e análise das atitudes em relação aos } \\
\text { idosos apresentada por seus } \\
\text { professores e estudantes }\end{array}$ & $\begin{array}{l}\text { Santos LLC, Bub } \\
\text { LIR, Mendes } \\
\text { NTC. }\end{array}$ & Revista Ciência e Saúde & 1990 & Pesquisa \\
\hline $\begin{array}{l}\text { Perceptions of elder abuse: voices } \\
\text { of professionals and } \\
\text { volunteers in Swiss - } \\
\text { an exploratory study }\end{array}$ & $\begin{array}{l}\text { Erlingsson CL, } \\
\text { Carison SL, Saveman } \\
\text { BI. }\end{array}$ & Scand J Caring Science & 2006 & Pesquisa \\
\hline $\begin{array}{l}\text { Soignants et bénévoles } \\
\text { en institution gériatrique: } \\
\text { quelle collaboration? }\end{array}$ & Guillot M. & Rech Soins Infirm & 1996 & Reflexão \\
\hline
\end{tabular}


Quadro 2 - Pesquisas de Enfermagem em Gerontologia. Curitiba, Paraná, 2007

Continuação

\begin{tabular}{|c|c|c|c|c|}
\hline Nome do Trabalho & Autor & Fonte & $\begin{array}{c}\text { Ano } \\
\text { publicação }\end{array}$ & $\begin{array}{l}\text { Tipo de } \\
\text { estudo }\end{array}$ \\
\hline $\begin{array}{l}\text { Difficult behaviors in long-term care patientes } \\
\text { with dementia }\end{array}$ & Buhr GT, White HK. & J Am Med Dir Assoc & 2006 & Pesquisa \\
\hline $\begin{array}{l}\text { Will older persons and their clinicians use a } \\
\text { shared decision-making instrument? }\end{array}$ & $\begin{array}{l}\text { Naik AD, Schulman-Green } \\
\text { D, McCorkie R, Bradley } \\
\text { EH, Bogardus S T. }\end{array}$ & J Gen Intern Med & 2005 & Pesquisa \\
\hline $\begin{array}{l}\text { Explorarion of patient's needs for information } \\
\text { on arrival at a geriatric and rehabilitation unit }\end{array}$ & $\begin{array}{l}\text { McKain S, Henderson A, } \\
\text { Kuys S, Drake S, } \\
\text { Kerridge L, Aher K. }\end{array}$ & J Clin Nurs & 2006 & Pesquisa \\
\hline $\begin{array}{l}\text { Healthcare providers' perspectives: estimating } \\
\text { the impact of chronicity }\end{array}$ & $\begin{array}{l}\text { Falkenstern SK, Loeb SJ. } \\
\text { Gueldner SH, Penrod J, } \\
\text { Poon LW. }\end{array}$ & $\begin{array}{l}\text { J Am Acad Nurs } \\
\text { Pract }\end{array}$ & 2005 & Pesquisa \\
\hline $\begin{array}{l}\text { A multifactorial intervention program reduces the } \\
\text { duration of delirium, length of hospitalization and } \\
\text { mortality in delirious patients }\end{array}$ & $\begin{array}{l}\text { Lundstrom M, Edlund A, } \\
\text { Karisson S, Brannstrom B, } \\
\text { Bucht G, Gustafson Y. }\end{array}$ & J Am Geriatr Soc & 2005 & Pesquisa \\
\hline $\begin{array}{l}\text { Feasible model for prevention of functional } \\
\text { deline in older people: municipality-randomized, } \\
\text { controlled trial }\end{array}$ & $\begin{array}{l}\text { Vass M, Avlund K, } \\
\text { Lauridsen J, Hendriksen C. }\end{array}$ & J Am Geriatric Soc & 2005 & Pesquisa \\
\hline $\begin{array}{l}\text { Interfenerational service-learning levels and } \\
\text { disciplines: [quot] one size (does not) fit all [quot] }\end{array}$ & $\begin{array}{l}\text { Karasik RJ, Maddox M, } \\
\text { Wallingfor DM. }\end{array}$ & Geronol Geriatr Educ & 2004 & Pesquisa \\
\hline $\begin{array}{l}\text { Care insurance and education, practice and } \\
\text { research of geriatric medicie }\end{array}$ & Ito $\mathrm{H}$. & $\begin{array}{l}\text { Nippon Ronen } \\
\text { Igakkai Zasshi }\end{array}$ & 2004 & Pesquisa \\
\hline $\begin{array}{l}\text { Assessments and nursing cate fr right brain- } \\
\text { damaged stroke patientd: focusing on neglect } \\
\text { and related symptoms }\end{array}$ & $\begin{array}{l}\text { Ohshima H, Murashima S, } \\
\text { Takahashi R. }\end{array}$ & Nurs Health Sci & 2004 & Pesquisa \\
\hline $\begin{array}{l}\text { Evidence-basead practice residency program in } \\
\text { gerontological nursing }\end{array}$ & Tang JH, Tiller MG. & J Gerontol Nurs & 2003 & Reflexão \\
\hline $\begin{array}{l}\text { From nursing home to hospital and back again: } \\
\text { an educational program to improve care for } \\
\text { hospitalized nursing home patients }\end{array}$ & Robinson SB, Weitzel T. & J Nurses Staff Dev & 2003 & Pesquisa \\
\hline $\begin{array}{l}\text { Attitudes toward working on interdisciplinary } \\
\text { healthcare teams: a comparison by discipline }\end{array}$ & $\begin{array}{l}\text { Leipzig RM, Hyer K, EK K, } \\
\text { Wallenstein S, Vezina ML, } \\
\text { Fairchild S, Cassel CK, } \\
\text { Howel JL. }\end{array}$ & J Am Geriatr Soc & 2002 & Reflexão \\
\hline Nursing and gerontology & McBride AB. & J Gerontolo Nurs & 2000 & Reflexão \\
\hline $\begin{array}{l}\text { The Hospital Elder Life Program: a model of } \\
\text { care to prevent cognitive and functional decline } \\
\text { in older hospitalized patients }\end{array}$ & $\begin{array}{l}\text { Inouye SK, Bogardus ST, } \\
\text { Baker DT, Leo-Summers } \\
\text { L, Cooney LM. }\end{array}$ & J Am Geriatr Soc & 2000 & Pesquisa \\
\hline Nursing research publications on older adults & Kovner CT, Mezey M. & Nurs Res & 2000 & Pesquisa \\
\hline $\begin{array}{l}\text { Geriatric patients with depresión. Improvin } \\
\text { outcomes using a multidisciplinary clinical path } \\
\text { model. }\end{array}$ & $\begin{array}{l}\text { Bultema JK, Mailliard L, } \\
\text { Getzfrid MK, Lerner RD, } \\
\text { Colone M. }\end{array}$ & J Nurs Adm & 1996 & Pesquisa \\
\hline
\end{tabular}


Observa-se no Quadro 2 que todos os estudos encontrados eram do tipo pesquisa ou reflexão e que os mesmos foram publicados no período de 1996 a 2007. Entretanto, 72\% das publicações datam após o ano de 2000, demonstrando maior interesse e/ou maior divulgação das produções de enfermagem em gerontologia neste período. Estes dados confirmam, aproximadamente, uma triplicação no número de produções, nos últimos seis anos, em relação à década anterior.

Corroborando os dados encontrados na presente pesquisa com os achados em estudos anteriormente publicados ${ }^{(10)}$, verifica-se que as pesquisas em enfermagem gerontológica e geriátrica no país caracterizam-se como irrisórias e que a veiculação da produção de conhecimento é restrita aos profissionais da prática. Sendo assim, preconiza-se a necessidade de incentivar a discussão das questões do envelhecimento. Constata-se que a proporção de trabalhos que envolvem a enfermagem, a pesquisa e a gerontologia apresentou um aumento considerável, porém ainda são poucos os editados no idioma português sobre esta temática.

Das produções encontradas, $65 \%$ estão no idioma inglês e publicadas em maior proporção por enfermeiras norte-americanas. Este fato sugere a discussão e análise sobre a relevância e necessidade de publicações, oriundas de pesquisas realizadas nas práticas da enfermagem brasileira, visto que esta tem características peculiares e diferenciadas das demais realidades.

O número de artigos, 26 (vinte e seis), demonstra o interesse sobre os assuntos abordados. Considera-se uma produção meritória, por se tratar de pesquisas que trazem subsídios essenciais ao trabalho da enfermagem gerontológica, beneficiando os profissionais e a comunidade acadêmica.

A análise documental quanto ao conteúdo temático das publicações encontradas possibilitou a identificação de cinco categorias temáticas principais, sendo que cinco trabalhos abordavam a geriatria e gerontologia na graduação; doze trabalhos relacionavam-se com a prática gerontológica; três publicações estavam relacionadas com a capacidade funcional de idosos institucionalizados; quatro artigos referendavam consulta de enfermagem gerontológica e/ou instrumentos de enfermagem para atenção ao idoso e três abordavam grupos de estudo e pesquisa em gerontologia.

Verifica-se que o tema com maior número de trabalhos se relaciona à prática profissional. Nessa, cabe salientar que significativo percentual é de produções norte-americanas e está relacionado às patologias que acometem os idosos, principalmente doenças crônicas.

O aumento da expectativa de vida e extensivo aumento do número de idosos na população ampliaram a proporção de portadores de doenças crônicas ${ }^{(11)}$. É significativa a proximidade dos profissionais nas atividades cotidianas com as patologias crônicas que acometem a maioria dos idosos, porquanto previsível esse interesse.

Empiricamente, constatamos a existência de número significativo de programas de pós-graduação stricto sensu e grupos de pesquisa em enfermagem gerontológica em nível nacional que representam fontes importantes de produção densa e periódica. Entretanto, esta investigação evidenciou a escassez de trabalhos brasileiros envolvendo a enfermagem e a gerontologia. Isso pode se justificar, em parte, pelo déficit de publicações em bases indexadas, ou ainda a problemas na indexação das palavras-chaves.

\section{CONSIDERAÇÕES FINAIS}

O estudo ressalta um maior número de publicações realizadas após o ano 2000, revelando o despertar das pesquisas envolvendo a enfermagem e a gerontologia, e o que traz mais alento é a constatação de que a maioria dos estudos teve como foco a prática profissional. Acredita-se que no final desta década as publicações na área gerontológica serão mais intensas, assim como a melhoria do modo de ordenação dessas na forma de índice, o que irá contribuir para a localização da informação referente à esse corpo de produção.

A área da gerontologia tem como característica marcante a multiplicidade de dimensões que a constitui e que ainda outras precisam ser reveladas por meio de estudos. Estes, por sua vez, possibilitam inovações imprescindíveis para o cotidiano profissional da enfermagem gerontológica e devem ser considerados como prioridade. Promover a disseminação do conhecimento da enfermagem gerontológica através da publicação é exigência para a expansão desta área do saber e para a fundamentação das ações profissionais específicas.

\section{REFERÊNCIAS}

1 Neri AL. Qualidade de vida na velhice. In: M. Delitti, organizador. Sobre comportamento e cognição: a prática da análise do comportamento e da terapia 
cognitivo-comportamental. Campinas: Arbytes; 1997.

2 Pessini L, Campos Junior O, Emim JAS. Pesquisa em saúde: reflexão sobre a interação com a realidade brasileira. Mundo Saúde. 2005;29(29):126.

3 Pádua EMM. Metodologia da pesquisa: abordagem teórico-prática. $9^{\mathrm{a}}$ ed. Campinas: Papirus; 2003.

4 Bardin L. Análise de conteúdo. Lisboa: Edições 70; 1977.

5 Neves EP, Souza IEO. Pesquisa em enfermagem: buscando resgatar a posição do sujeito que a desenvolve. Texto Contexto Enferm. 2003;12(3):387-93.

6 Padilha MICS. Editorial. Texto Contexto Enferm. 2003; 12(3):269.

7 Leopardi MT. Fundamentos gerais da produção científica. In: Leopardi MT. Metodologia da pesquisa em saúde. $2^{\mathrm{a}}$ ed. Florianópolis: UFSC; 2002.

8 Silva EBNS, Brêtas ACP. Grupos de estudo e pesquisa em gerontologia que incluem a enfermagem. Texto Contexto Enferm. 1997;6(2):106-17.

9 Lenardt MH, Willig MH, Silva SC, Shimbo AY, Tallmann AEC, Maruo GH. O idoso institucionalizado e a cultura de cuidados profissionais. Cogitare Enferm. 2006;11(2):117-23.

10 Mendes MMR, Rodrigues RAP, Oliveira CCL, Kusumota L. Pesquisa em enfermagem geriátrica e gerontológica. Texto Contexto Enferm. 1997;6:58-68.

11 Barreto NDM. Diabetes mellitus na pessoa idosa. Arq Geriatr Gerontol. 1997;(1):65-71. 